\title{
Isometry of medial collateral ligament reconstruction
}

\author{
Brian T. Feeley $\cdot$ Mark S. Muller • \\ Answorth A. Allen · Carinne C. Granchi · \\ Andrew D. Pearle
}

Received: 14 January 2009/ Accepted: 31 March 2009/Published online: 7 May 2009

(c) The Author(s) 2009. This article is published with open access at Springerlink.com

\begin{abstract}
The purpose of this study was to determine the femoral and tibial fixation sites that would result in the most isometric MCL reconstruction technique. Seven cadaveric knees were used in this study. A navigation system was utilized to determine graft isometry continuously from $0^{\circ}$ to $90^{\circ}$. Five points on the medial side of the femur and four on the tibia were tested. A graft positioned in the center of the MCL femoral attachment $\left(\mathrm{F}_{\mathrm{C}}\right)$ and attached in the center of the superficial MCL attachment on the tibia led to the best isometry $(2.7 \pm 1.1 \mathrm{~mm})$. Movement of the origin superiorly only $4 \mathrm{~mm}\left(\mathrm{~F}_{\mathrm{S}}\right)$ led to graft excursion of greater than $10 \mathrm{~mm}(P<0.01)$. MCL reconstruction performed with the origin of the MCL within the femoral footprint and the insertion in tibial footprint of the superficial MCL results in the least graft excursion when the knee is cycled between $0^{\circ}$ and $90^{\circ}$. Although the MCL often heals without surgical intervention, surgical reconstruction is occasionally in Grade III MCL and combined ligamentous injuries to the knee. This study demonstrates
\end{abstract}

B. T. Feeley $(\bowtie)$

Department of Orthopaedic Surgery, University of California, UCSF, 1701 Divisadero Street, Suite 240,

San Francisco, CA 94115, USA

e-mail: feeleyb@orthosurg.ucsf.edu

M. S. Muller

Carroll Clinic, Dallas, TX, USA

A. A. Allen · A. D. Pearle

Sports Medicine and Shoulder Service,

Department of Orthopaedic Surgery,

Hospital for Special Surgery,

New York, NY 10021, USA

C. C. Granchi

Surgetics Institute, Praxim Medivision, Grenoble, France the optimal position of the MCL reconstruction to reproduce the kinematics of the native knee.

Keywords Superficial medial collateral ligament . Isometry $\cdot$ Reconstruction $\cdot$ Knee

\section{Introduction}

The medial side of the knee contains static and dynamic stabilizers that contribute to resisting valgus and rotational forces. The medial collateral ligament (MCL) is the primary stabilizer against valgus stress, especially at lower flexion angles. At $25^{\circ}$ of flexion, the MCL provides $78 \%$ of the restraining force against valgus injury [1]. At full extension, the MCL provides $57 \%$ of the restraining force, with the posterior oblique ligament (POL), ACL, and posteromedial capsule responsible for the majority of the remaining restraint. The MCL comprises two distinct structures: the superficial and deep MCL. The superficial MCL (sMCL) originates on an average of $3.2 \mathrm{~mm}$ proximal and $4.8 \mathrm{~mm}$ posterior to the medial epicondyle [2]. It inserts at two distinct points on the proximal medial tibia, just anterior to the posteromedial crest of the tibia. The deep portion of the MCL originates inferior to the medial epicondyle and inserts $1 \mathrm{~cm}$ below the joint line on the tibia.

Injuries to the medial structures of the knee are very common, particularly in contact and pivoting sports such as American football and soccer [3-5]. Despite the frequency of these injuries, successful treatment can be achieved with non-operative management in a majority of MCL injuries. Similar results have been found with both operative and non-operative management of these injuries [6]. Athletes with combined ACL-MCL injuries can expect acceptable stability and a good to excellent functional outcome with 
isolated ACL reconstruction alone [7, 8]. However, knee dislocations with injury to both the medial and lateral side of the knee may require superficial MCL reconstruction in up to $85 \%$ of patients [9]. There are also a small but significant number of patients who continue to have residual symptoms of pain, instability, or both following an MCL injury and require surgical reconstruction [10].

There have been numerous techniques described for superficial MCL reconstruction, many of which do not attempt to reconstruct the native anatomy of the medial side of the knee. The original reconstruction technique described by Bosworth was a non-anatomic reconstruction using the semi-tendonosus tendon [11]. The maintenance of graft isometry or constant length during knee range of motion has been advocated as an important goal in knee ligament reconstruction $[12,13]$.

The purpose of this study was to determine the femoral and tibial fixation sites that would result in the most isometric superficial MCL reconstruction technique. We also wished to determine at which flexion angles the grafts should be fixed in order to best restore knee stability. We hypothesized that an anatomic reconstruction that better replicated the native knee anatomy would result in the most isometric reconstruction. We used computer assisted navigation to determine graft length and isometry because it has previously been found to be a precise biomechanics tool to measure graft isometry $[12,14]$.

\section{Methods}

Seven fresh-frozen cadaveric knees that had been used in a previous study were used in the current study. The knees had an average age of 51.3 years. Five were male knees, two were female. Knees were excluded from the study if there was ligamentous laxity, significant arthritic changes, gross malalignment, or evidence of previous surgery. The tibia and femur were sectioned at the mid-shaft and soft tissue was removed up to $15 \mathrm{~cm}$ from the joint line. The specimens were mounted and secured using a vice attached to the proximal femur. The knees were mounted to allow free range of motion from $0^{\circ}$ to $110^{\circ}$ of flexion.

The medial side of the knee was dissected and exposed to visualize the MCL. The femoral attachment of the superficial MCL was identified and carefully dissected free from its proximal insertion. The attachment of the superficial MCL on the proximal medial tibia was similarly dissected and marked to identify the proximal and distal insertions of the sMCL. The deep MCL was then identified and its proximal and distal attachments were identified. The semitendinosus tendon was then identified in the pes anserinus and its insertion was dissected and marked accordingly. An isometric map was then generated with multiple points on the medial distal femur and the proximal tibia (Fig. 1). The distal femoral points corresponded to the femoral attachment of the MCL $\left(\mathrm{F}_{\mathrm{C}}\right)$, and $4 \mathrm{~mm}$ superior $\left(F_{S}\right)$, inferior $\left(F_{I}\right)$, anterior $\left(F_{A}\right)$, and posterior $\left(F_{P}\right)$ to the origin. There were four points registered on the tibia: one that corresponded to the anterior proximal $\left(\mathrm{T}_{\mathrm{P}}\right)$ aspect of the proximal attachment of the sMCL, one for the distal attachment of the sMCL $\left(\mathrm{T}_{\mathrm{D}}\right)$, one at the midpoint between the proximal and distal attachment $\left(\mathrm{T}_{\mathrm{M}}\right)$, and one for the insertion of the semitendonosus $\left(\mathrm{T}_{\mathrm{H}}\right)$.

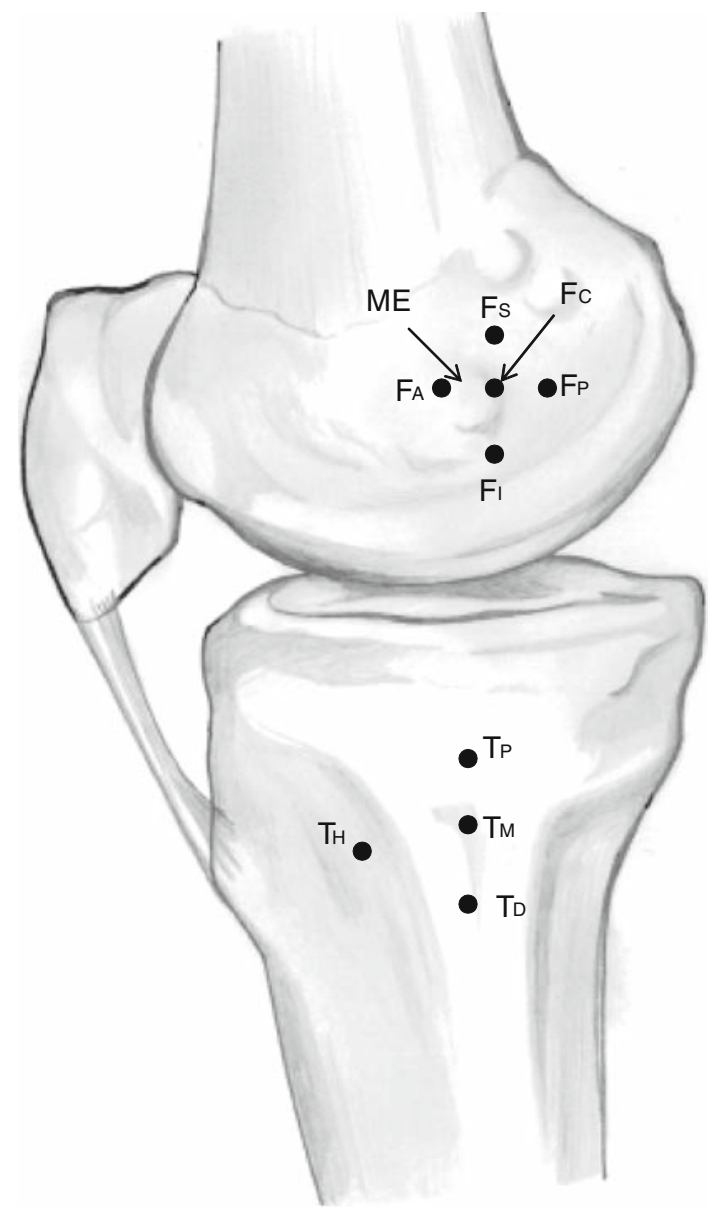

Fig. 1 Schematic of each point taken for isometry measurments. There were five points taken on the femur, based on the femoral attachment of the superficial MCL (Center MCL). A separate measurement point was created $4 \mathrm{~mm}$ in each direction (anterior, posterior, superior, and inferior) from the center of the MCL femoral attachment. On the tibial side, four points were again identified. One point was at the superior tibial attachment of the superficial MCL. One point was at the distal insertion of the sMCL. One point was at the midpoint of the sMCL attachment. Finally, one was at the semitendonosus insertion. $M E$ Medial epicondyle; $F_{S}$ Superior femoral attachment of the MCL; $F_{C}$ Center of sMCL attachment on femur; $F_{I}$ Interior femoral attachment; $F_{P}$ Posterior femoral attachment; $F_{A}$ Anterior femoral attachment; $T_{M}$ Midpoint of the attachment of the sMCL on the tibia; $T_{H}$ Semitendinosus insertion on tibia; $T_{P}$ Proximal aspect of sMCL attachment on tibia; $T_{A}$ Anterior aspect of sMCL attachment on tibia; $T_{D}$ Distal aspect of sMCL attachment on tibia. Figure courtesy of Joseph Smith, ATC 
The Praxim Surgetics surgical navigation system (PraximMedivision, Grenoble, France) was used to acquire isometric data. The Surgetics ACL Logics Universal Software was used for data acquisition as previously described [14]. The technology generated a three dimensional image of the articular anatomy by acquiring reference points directly on the bony surfaces and then forming a statistical model to fit these points. The reference points included the center of the femoral notch, the middle of the transverse meniscal ligament on the anterior tibia, and the center of the medial and lateral tibial plateaus. This system has been shown to be precise; within $1^{\circ}$ or $1 \mathrm{~mm}$ compared with an industrial robotic sensor [14-16]. Threaded Steinmann pins were placed in the proximal femur and distal tibia to mount the reflective markers. Surface landmarks on the tibial plateau and distal femur were recorded, intraarticular surface geometry was mapped, and the 3-D model was created. The knee was manually cycled from full extension to $110^{\circ}$ of flexion.

The isometry of each graft reconstruction, defined as the change in graft length through a given range of motion, was determined at continuous intervals from $0^{\circ}$ to $90^{\circ}$ of knee flexion as recorded by the navigation software. Fiber lengths were normalized to zero at full extension. The length change between the fiber length at full extension and the fiber length at the respective flexion angle was computed at $5^{\circ}$ increments from $0^{\circ}$ to $90^{\circ}$. These length changes were subsequently plotted to demonstrate the anisometry 'profile' of each fixation point from $0^{\circ}$ to $90^{\circ}$. The total length change in each fiber was also computed, as was the strain in the fiber $(\Delta \mathrm{L} / \mathrm{L})$.

Table 1 Isometry of graft positions

\begin{tabular}{llllll}
\hline \multicolumn{5}{l}{ Femoral position } \\
\cline { 2 - 6 } & $\mathrm{F}_{\mathrm{C}}(\mathrm{mm})$ & $\mathrm{F}_{\mathrm{A}}(\mathrm{mm})$ & $\mathrm{F}_{\mathrm{P}}(\mathrm{mm})$ & $\mathrm{F}_{\mathrm{S}}(\mathrm{mm})$ & $\mathrm{F}_{\mathrm{I}}(\mathrm{mm})$ \\
\hline $\mathrm{T}_{\mathrm{P}}$ & $2.7 \pm 1.2$ & $3.1 \pm 1.0$ & $7.2 \pm 2.7^{\mathrm{a}}$ & $6.5 \pm 1.2^{\mathrm{a}}$ & $9.8 \pm 2.9^{\mathrm{a}}$ \\
$\mathrm{T}_{\mathrm{M}}$ & $3.1 \pm 1.7$ & $4.3 \pm 1.5$ & $6.0 \pm 1.5^{\mathrm{a}}$ & $8.3 \pm 2.0^{\mathrm{a}}$ & $8.4 \pm 3.1^{\mathrm{a}}$ \\
$\mathrm{T}_{\mathrm{D}}$ & $2.8 \pm 1.1$ & $3.2 \pm 1.5$ & $7.2 \pm 1.5^{\mathrm{a}}$ & $6.7 \pm 2.1^{\mathrm{a}}$ & $9.7 \pm 2.7^{\mathrm{a}}$ \\
$\mathrm{T}_{\mathrm{H}}$ & $4.1 \pm 2.3^{\mathrm{a}}$ & $6.1 \pm 2.2^{\mathrm{a}}$ & $4.6 \pm 2.3^{\mathrm{a}}$ & $10.8 \pm 3.1^{\mathrm{a}}$ & $5.1 \pm 3.0$ \\
\hline $\mathrm{a} P<0.05$ compared to proximal insertion and center of MCL (most \\
isometric position)
\end{tabular}

Repeated measures ANOVA was used to compare the anisometry of the grafts at the various flexion angles. Significance was defined as a $P$ value less than 0.05 . Data are expressed as the mean \pm standard error of the mean (SEM).

\section{Results}

The isometric data are summarized in Table 1. Proximally, placement of the graft at the center of the superficial MCL attachment on the femur resulted in the best overall graft isometry. The mean change in length from the center of the MCL to the proximal $\left(\mathrm{T}_{\mathrm{P}}\right)$ and distal tibial insertions $\left(\mathrm{T}_{\mathrm{D}}\right)$ of the MCL on the tibia were $2.7 \pm 1.2 \mathrm{~mm}$ and $2.8 \pm 1.1 \mathrm{~mm}$, respectively. There was no significant difference in length change with placement of the graft on the anterior aspect of the tibial attachment $\left(\mathrm{T}_{\mathrm{A}}\right)$ or at the midpoint of the attachment $\left(\mathrm{T}_{\mathrm{M}}\right)$. However, the mean change in length was significantly greater with placement of the graft anteriorly at the site of the semitendonosus insertion $\left(\mathrm{T}_{\mathrm{H}}\right)$ (4.1士, $P<0.05$ ) which would correspond to the placement of the anterior limb of the modified Bosworth technique.

Movement of the graft anteriorly $4 \mathrm{~mm}\left(\mathrm{~F}_{\mathrm{A}}\right)$ from the origin of the sMCL resulted in a greater mean change of length with the graft fixed distally at the anterior aspect of the insertion as well as at the hamstring insertion (Table 1). Posterior fixation $\left(\mathrm{F}_{\mathrm{P}}\right)$ of the graft led to significant increases in mean length change at all points within the MCL insertion. Similarly, fixation of the graft at either $F_{S}$ or $F_{I}$ led to greater anisometry with each point tested on the tibia. The greatest anisometry was seen from $F_{S}$ to $T_{H}$ (10.8 $\pm 3.1 \mathrm{~mm}, P<0.01$ vs. $\mathrm{F}_{\mathrm{C}}$ to all tibial points).

The strain measurements are reported in Table 2. The overall mean strain measurements were low, with none reaching greater than 0.11 . The grafts positioned in the points that corresponded to the most anatomic reconstruction $\left(\mathrm{F}_{\mathrm{C}}\right.$ to $\mathrm{T}_{\mathrm{P}}$ and $\left.\mathrm{T}_{\mathrm{D}}\right)$ had the lowest strain $(\varepsilon=0.02 \pm 0.005)$. The strain was greatest in the least isometric positions, especially from the $\mathrm{F}_{\mathrm{S}}$ to $\mathrm{T}_{\mathrm{H}}(\varepsilon=0.12 \pm 0.04)$.

The absolute graft lengths were longest with the knee in full extension, regardless of the fixation points selected. As the knee was flexed to $90^{\circ}$, the absolute graft lengths shortened with all fixation configurations. A majority of the shortening occurred from 0 to $30^{\circ}$, suggesting that the graft
Table 2 Strain $(\varepsilon)$ of each graft position

a $P<0.05$ compared to proximal insertion and center of MCL (most isometric position)

\begin{tabular}{|c|c|c|c|c|c|}
\hline & \multicolumn{5}{|c|}{ Femoral position } \\
\hline & $\mathrm{F}_{\mathrm{C}}$ & $\mathrm{F}_{\mathrm{A}}$ & $\mathrm{F}_{\mathrm{P}}$ & $\mathrm{F}_{\mathrm{S}}$ & $\mathrm{F}_{\mathrm{I}}$ \\
\hline $\mathrm{T}_{\mathrm{A}}$ & $0.02 \pm 0.003$ & $0.03 \pm 0.003$ & $0.06 \pm 0.01$ & $0.06 \pm 0.011$ & $0.09 \pm 0.017^{\mathrm{a}}$ \\
\hline $\mathrm{T}_{\mathrm{M}}$ & $0.03 \pm 0.008$ & $0.03 \pm 0.004$ & $0.04 \pm 0.012$ & $0.06 \pm 0.013$ & $0.06 \pm 0.009$ \\
\hline $\mathrm{T}_{\mathrm{D}}$ & $0.02 \pm 0.007$ & $0.03 \pm 0.006$ & $0.06 \pm 0.012$ & $0.06 \pm 0.018$ & $0.08 \pm 0.031^{\mathrm{a}}$ \\
\hline $\mathrm{T}_{\mathrm{H}}$ & $0.03 \pm 0.005$ & $0.05 \pm 0.010$ & $0.04 \pm 0.007$ & $0.11 \pm 0.031^{\mathrm{a}}$ & $0.04 \pm 0.01$ \\
\hline
\end{tabular}




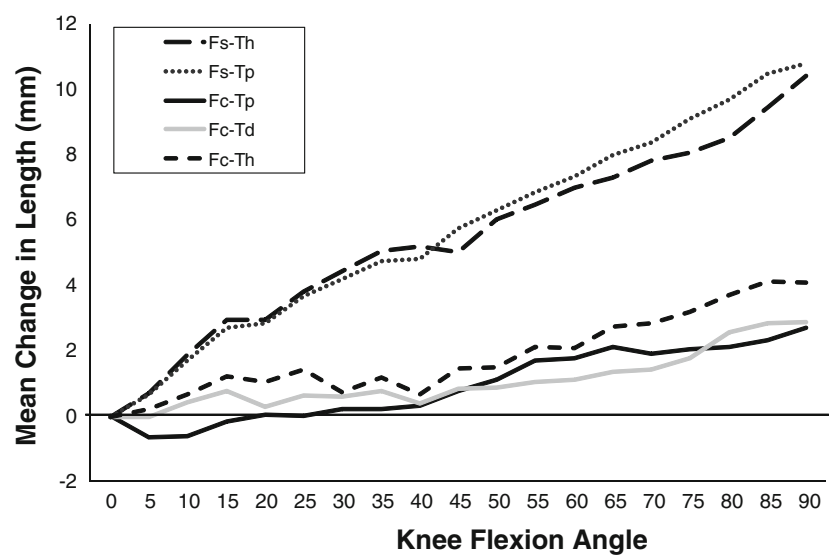

Fig. 2 Mean relative length changes of select MCL reconstruction techniques. The values are references to the value of the absolute graft length at $0^{\circ}$ of flexion. Positive numbers reflect graft shortening as the knee is flexed toward $90^{\circ}$. Negative numbers reflect graft lengthening. $F_{S}$ Superior femoral origin; $F_{C}$ Center of MCL origin on femur. $T_{H}$ Hamstring insertion on tibia; $T_{P}$ Proximal aspect of MCL insertion on tibia; $T_{A}$ Anterior aspect of MCL insertion on tibia; $T_{D}$ Distal aspect of MCL insertion on tibia

should be tensioned at lower flexion angles to avoid over constraint of the knee as the knee is flexed. Representative graft isometry graphs demonstrating the most isometric and least isometric configurations are shown in Fig. 2.

\section{Discussion}

In this study, we found that the ideal isometric reconstruction of the superficial MCL was with the graft originating at the anatomic femoral attachment of the sMCL and was placed on the proximal and distal attachments of the sMCL on the tibia. This position also led to the least strain on the graft.

The optimal isometric position of the MCL reconstruction on the femur was with the graft placed at the femoral attachment of the sMCL. Placement of the graft $4 \mathrm{~mm}$ in any direction away from the sMCL origin resulted in significant increases in graft length during knee range of motion, suggesting that precise anatomic placement of the graft within this region is important in maintaining knee stability following reconstruction. In this study, great care was taken to identify the anatomic location of the origin of the sMCL as it related to the medial epicondyle. Our data were similar to data reported by LaPrade et al. [2]. We found that the sMCL attaches to the femur just proximal and posterior to the medial epicondyle. Although there is some variability between samples, all sMCL attachments were within $5 \mathrm{~mm}$ proximal and posterior to the medial epicondyle, suggesting this is a reliable landmark to reproduce the femoral attachment of the sMCL during reconstruction.
Distally, the most isometric points of graft fixation were with the graft fixed in the proximal or distal aspects of the sMCL insertion on the medial aspect of the tibia. Fixation of the graft anteriorly in the semitendonosus insertion led to significantly greater changes in graft length. These data have several important clinical implications. First, many surgeons prefer to use a modification of the Bosworth technique where the semitendonosus tendon is left intact at the insertion, attached proximally on the medial side of the femur, and attached distally in the MCL footprint. This study suggests that fixation with this technique leads to a very anisometric reconstruction and may result in clinical laxity over time. However, the calculated strain with the graft placed anteriorly in the semitendonosus footprint was only minimally greater than when placed in the MCL footprint. A second important clinical finding was that there were two optimal isometric points within the insertion of the sMCL. Recent studies in our lab have found that an anatomic double bundle MCL reconstruction best restored native knee laxity patterns [17]. Fixation of a double bundle graft both proximally and distally within the footprint of the MCL would lead to a reconstruction with good overall isometry.

A majority of the studies on graft isometry has been performed on cruciate ligament reconstructions, and only recently have studies evaluated graft isometry on the posterolateral corner [18]. Graft isometry is important for any ligament reconstruction in order to effectively stabilize the joint during the entire range of motion. This study demonstrates that the relative lengths of MCL grafts decrease with knee flexion, and most of this length decrease occurs from $45^{\circ}$ to $90^{\circ}$. If a graft was tensioned with the knee flexed to $90^{\circ}$, it would result in a graft that would be excessively tightened as the knee was extended to $0^{\circ}$. The excessive tension in the graft could lead to excessive graft forces which can cause the graft to permanently stretch or even fail during the early healing period. Conversely, if the graft was fixed at or close to full extension, the graft would slacken slightly and not over constrain the knee as it was flexed toward $90^{\circ}$. Thus, any MCL reconstruction should be tensioned at or close to full extension.

There are several weaknesses to this study. The use of computer assisted navigation to evaluate isometry has only recently been utilized, and the technology has not been validated with independent studies. Previous studies on our lab have used this technique to accurately determine ACL isometry that was consistent with earlier published data [12]. In addition most previous isometric studies have used single sutures to measure graft length changes, which may not account for differential isometry within a 7-9 mm graft. The computer assisted navigation system that we utilize averages the isometry for a proposed graft, which may be more precise than using a suture technique to 
measure graft length change. Studies comparing the two techniques would be beneficial. A second weakness of this study is that although it found significant differences in graft isometry, it cannot answer whether these changes would be clinically relevant, especially in light of the fact that there were only small changes in the strain seen with each graft configuration. Clinical studies evaluating the outcomes with grafts in different configurations would help determine if small changes in graft position truly result in different clinical outcomes.

\section{Conclusion}

Our results suggest that sMCL reconstruction performed with the femoral attachment of the MCL within the femoral footprint and the tibial attachment within the footprint of the sMCL will result in the least graft excursion when the knee is cycled between $0^{\circ}$ and $90^{\circ}$. Graft fixation should be performed at or near full extension in order to not over constrain the knee or overload the MCL reconstruction, which could lead to early failure of the reconstruction.

Acknowledgment Figure courtesy of Joseph Smith, ATC was acknowledged.

Open Access This article is distributed under the terms of the Creative Commons Attribution Noncommercial License which permits any noncommercial use, distribution, and reproduction in any medium, provided the original author(s) and source are credited.

\section{References}

1. Grood ES, Noyes FR, Butler DL et al (1981) Ligamentous and capsular restraints preventing straight medial and lateral laxity in intact human cadaver knees. J Bone Joint Surg Am 63:1257-1269

2. LaPrade RF, Engebretsen AH, Ly TV et al (2007) The anatomy of the medial part of the knee. J Bone Joint Surg Am 89:20002010. doi: 10.2106/JBJS.F.01176

3. Adickes MS, Stuart MJ (2004) Youth football injuries. Sports Med 34:201-207. doi:10.2165/00007256-200434030-00005

4. Brophy RH, Barnes R, Rodeo SA et al (2007) Prevalence of musculoskeletal disorders at the NFL Combine-trends from
1987 to 2000. Med Sci Sports Exerc 39:22-27. doi:10.1249/01. mss.0000241637.52231.18

5. Larsen G, Jensen PK, Jensen PR (1999) Long-term outcome of knee and ankle injuries in elite football. Scand J Med Sci Sports 9:285-289

6. Indelicato PA (1983) Nonoperative treatment of complete tears of the medial collateral ligament of the knee. J Bone Joint Surg Am 65:323-329

7. Noyes FR, Barber-Westin SD (1995) The treatment of acute combined ruptures of the anterior cruciate and medial ligaments of the knee. Am J Sports Med 23:380-389. doi:10.1177/ 036354659502300402

8. Shelbourne KD, Porter DA (1992) Anterior cruciate ligamentmedial collateral ligament injury: nonoperative management of the medial collateral ligament tears with anterior cruciate ligament reconstruction - a preliminary report. Am J Sports Med 20:283-286. doi:10.1177/036354659202000308

9. Cooper DE, Stewart D (2004) Posterior cruciate ligament reconstruction using single-bundle patella tendon graft with tibial inlay fixation: 2- to 10-year follow-up. Am J Sports Med 32:346360. doi:10.1177/0363546503261511

10. Lonergan KT, Taylor DC (2002) Medial collateral ligament injuries of the knee: an evolution of surgical reconstruction. Tech Knee Surg 1:137-145

11. Bosworth DM (1952) Transplantation of the semitendinosus for repair of laceration of medial collateral ligament of the knee. J Bone Joint Surg Am 34-A:196-202

12. Pearle AD, Shannon FJ, Granchi C et al (2008) Comparison of 3dimensional obliquity and anisometric characteristics of anterior cruciate ligament graft positions using surgical navigation. Am J Sports Med 36:1534-1541. doi:10.1177/0363546508315536

13. Penner DA, Daniel DM, Wood P et al (1988) An in vitro study of anterior cruciate ligament graft placement and isometry. Am J Sports Med 16:238-243. doi:10.1177/036354658801600307

14. Pearle AD, Solomon DJ, Wanich T et al (2007) Reliability of navigated knee stability examination: a cadaveric evaluation. Am J Sports Med 35:1315-1320. doi:10.1177/0363546507300821

15. Hufner T, Geerling J, Kfuri M Jr et al (2003) Computer-assisted pelvic surgery: registration based on a modified external fixator. Comput Aided Surg 8:192-197. doi:10.3109/10929080309146053

16. Khadem R, Yeh CC, Sadeghi-Tehrani M et al (2000) Comparative tracking error analysis of five different optical tracking systems. Comput Aided Surg 5:98-107. doi:10.3109/10929080009148876

17. Feeley BT, Muller MS, Granchi C et al. (2009) Biomechanical comparison of medial collateral ligament reconstructions using computer assisted navigation. Am J Sports Med (in press)

18. Sigward SM, Markolf KL, Graves BR et al (2007) Femoral fixation sites for optimum isometry of posterolateral reconstruction. J Bone Joint Surg Am 89:2359-2368. doi:10.2106/JBJS.F.01132 